\title{
Bipartite entanglement of quantum states in a pair basis
}

\author{
Marco Roncaglia, ${ }^{1,2}$ Arianna Montorsi, ${ }^{1}$ and Marco Genovese ${ }^{2}$ \\ ${ }^{1}$ Dipartimento di Fisica del Politecnico, corso Duca degli Abruzzi 24, 10129 Torino, Italy \\ ${ }^{2}$ INRIM, strada delle Cacce 91, 10135 Torino, Italy
}

(Dated: September 4, 2018)

\begin{abstract}
The unambiguous detection and quantification of entanglement is a hot topic of scientific research, though it is limited to low dimensions or specific classes of states. Here we identify an additional class of quantum states, for which bipartite entanglement measures can be efficiently computed, providing new rigorous results. Such states are written in arbitrary $d \times d$ dimensions, where each basis state in the subsystem A is paired with only one state in B. This new class, that we refer to as pair basis states, is remarkably relevant in many physical situations, including quantum optics. We find that negativity is a necessary and sufficient measure of entanglement for mixtures of states written in the same pair basis. We also provide analytical expressions for a tight lower-bound estimation of the entanglement of formation, a central quantity in quantum information.
\end{abstract}

PACS numbers: 03.67.Mn, 03.65.Ud, 42.65.Lm

Quantum entanglement, after having been considered for many years a peculiar aspect of quantum mechanics whose interest was limited to specialists in foundations in quantum mechanics [1, has assumed a pervasive role in contemporary science with applications that range from cosmology [2] to biology [3]. In particular it attracts a considerable interest being a fundamental resource for quantum technologies [1, 4, 7]. This widespread relevance prompted the need of its unambiguous detection and quantification, a result still largely unachieved [8, 9] and recently subject to a large theoretical effort [10]. Indeed, while bipartite entanglement in a pure state can be estimated using the von Neumann entropy as well as other measures, the problem of its evaluation is still open in the case of a general mixed state. A significant step forward has been done with the proof that that the bipartite entanglement in a general mixed state of a system of dimension $2 \times 2$ is suitably quantified by the concurrence 11. In $d \times d$ dimensions, with $d \geq 3$ [12, a suitable measure has not yet been found except in the presence of special symmetries, like in the case of Werner states [13. A computable entanglement monotone in arbitrary dimension is the negativity [14, which can be evaluated for different physical systems [15-17, but in general it represents only a sufficient condition for entanglement.

From the perspective of quantum information, it is helpful to evaluate the entanglement of formation (EOF), a faithful measure that quantifies the minimal entanglement resources needed to prepare a given state, in terms of Bell pairs. At variance with the negativity, the EOF is typically hard to calculate, as it is obtained from an optimization problem that only in some special cases can be solved analytically 11. Sometimes, in higher dimensional system (when exact EOF is impractical to evaluate), it is convenient to determine a lower bound to EOF which sets the amount of resources that are at least present inside the quantum state in consideration. Of course, such a lower bound should be as tight as possible, in order to be useful.

We define the set of pure pair basis states of a bipartite system in arbitrary dimension $d \times d$ as the set of states of the form

$$
|\Psi\rangle=\sum_{i=1}^{d} c_{i}\left|\phi_{i}\right\rangle_{A} \otimes\left|\chi_{i}\right\rangle_{B}
$$

where $\left\{\phi_{i}\right\}_{i=1}^{d}$ and $\left\{\chi_{i}\right\}_{i=1}^{d}$ are fixed orthonormal basis for Hilbert spaces of part $A$ and $B$, respectively. The coefficients $c_{i} \in \mathbb{C}$ satisfy the normalization condition $\sum_{i}\left|c_{i}\right|^{2}=1$. Of course, every pure state can be expressed in the form (1), through the Schmidt decomposition and arbitrary assignment of phase factors to every $c_{i}$. The peculiarity of this set is that all the states belonging to it share the same basis, where each element $\phi_{i}$ in $A$ is paired with only one $\chi_{i}$ in $B$. This represents a subset of the most general bipartite case, where quantum states of a given ensemble may have different (pair) basis after Schmidt decomposition.

In this paper, we address the problem of evaluating the entanglement of mixtures of states written in a pair basis (1), identifying suitable measures. Thus, we estimate rigorous and numerical lower bounds to the EOF, comparing them to other estimates proposed in the literature [18].

Pair basis states occur in a variety of physical situations, the most remarkable one being represented by two-mode Gaussian states in quantum optics [1, 19 21], that include twin-beam states, a key element of quantum communication, metrology and sensing. More interestingly, also non-Gaussian states are included in the family of states of Eq.(1), like for example a twin beam plus a dephased component or mixtures of photon subtracted states. For these latter cases, that are relevant in many modern experiments of quantum mechanics [22], good measures of entanglement have not been found, so far. Moreover, pair basis states are a natural way to explore high-dimensional entanglement, recently observed in spatial modes of pairs of down-converted photons [23. In atomic physics, bosonic atoms trapped in double wells have the same structure (1) where the conservation of the 
total number $N$ imposes that each state $|n\rangle$ of $n$ bosons in one well is paired with the state $|N-n\rangle$ in the other well. Also for electron models in a lattice may be interesting to restrict the total Hilbert space of two-sites to the pair basis $\{|\uparrow, \downarrow\rangle,|\downarrow, \uparrow\rangle,|\downarrow \uparrow, 0\rangle,|0, \downarrow \uparrow\rangle\}$, i.e. the sector of zero magnetization and two electrons, thanks to the presence of special quantum numbers.

\section{PURE STATES}

Given the orthonormality of both bases $\left|\phi_{i}\right\rangle_{A}$ and $\left|\chi_{i}\right\rangle_{B}$, the Schmidt decomposition of states of the form (1) is just written in the same basis, but with the non negative coefficients $\mu_{i}=\left|c_{i}\right|$. The Schmidt coefficients $\mu_{i}$ are the square roots of the eigenvalues of the reduced density matrix $\rho_{A}=\operatorname{Tr}_{B} \rho$. It follows that the only factorized states are those with $c_{i}=0$ for every $i$ except one. The entanglement of a pure state $|\Psi\rangle$ is estimated by the von Neumann entropy $S^{(d)}(\Psi)=-\sum_{i=1}^{d} \mu_{i}^{2} \log \mu_{i}^{2}$. In the following, we shorten $\left|\phi_{i}\right\rangle_{A} \otimes\left|\chi_{i}\right\rangle_{B} \equiv|i, i\rangle$.

For estimating the entanglement of pure states, in this work we use a generalization of the concurrence that we construct in the following way. First, let us consider the easiest case $d=2$ with the two elements of the basis $|0,0\rangle$ and $|1,1\rangle$. In the full 4-dimensional space the double spin-flip operation is performed by $\sigma_{A}^{y} \otimes \sigma_{B}^{y}$, and the concurrence is given by the well-known formula $C=\left|\left\langle\Psi\left|\sigma_{A}^{y} \otimes \sigma_{B}^{y}\right| \Psi^{*}\right\rangle\right|$, where $\Psi^{*}$ is the complex conjugate of $\Psi$ and $\sigma^{\alpha}, \alpha=x, y, z$ are the usual Pauli matrices acting on single qubits. In our special $d=2$ case for states in a pair basis, the concurrence becomes $C=\left|\left\langle\Psi\left|\tau^{x}\right| \Psi^{*}\right\rangle\right|=2\left|c_{1} c_{2}\right|$, where the swap is represented by the single Pauli matrix $\tau^{x}$ acting on the pair basis.

Any extension to the $d$-dimensional case has to take into account the fact that for product states, the concurrence has to be zero in every possible two-dimensional subspace. Even if the literature proposes different generalizations of the concurrence [24], we opt to take the sum over every two-dimensional concurrence

$$
D(\Psi)=2 \sum_{i<j}\left|c_{i} c_{j}\right|=\left(\operatorname{Tr} \sqrt{\rho_{A}}\right)^{2}-1 .
$$

which manifestly vanishes only in the factorized case and likewise it fulfills the additivity property whenever the dimension $d$ is built up by the direct product of twodimensional states. In our picture, each term of Eq. (2) considers the state $|j\rangle$ as the spin-flipped of $|i\rangle$. A remarkable fact that further justifies the choice of the quantity $D(\Psi)$ is that it turns out to be twice the negativity $\mathcal{N}(\rho) \equiv\left(\left\|\rho^{T_{A}}\right\|_{1}-1\right) / 2$, where $\rho^{T_{A}}$ stands for the partial transpose with respect to subsystem $A$ and $\|G\|_{1}=$ $\operatorname{Tr} \sqrt{G G^{\dagger}}$ is the trace norm. For proving this statement we write the entries of the density matrix of the state (1) in the full Hilbert space $\left\langle i, j|\rho| i^{\prime}, j^{\prime}\right\rangle=\delta_{i j} \delta_{i^{\prime} j^{\prime}} c_{i} c_{i^{\prime}}^{*}$. As a matter of fact, the operation of partial transposition introduces non vanishing matrix elements outside the set of pair basis states, obtaining $\left\langle i, j\left|\rho^{T_{A}}\right| i^{\prime}, j^{\prime}\right\rangle=\delta_{i j^{\prime}} \delta_{i^{\prime} j} c_{i} c_{i^{\prime}}^{*}$. Besides the diagonal part of $\rho$ that is left unchanged, the matrix $\rho^{T_{A}}$ displays $2 \times 2$ blocks for every pair $i<j$ in the subspace formed by the two basis vectors $|i, j\rangle$ and $|j, i\rangle$. The eigenvalues of such blocks turn out to be pairs of opposite numbers $\pm\left|c_{i} c_{j}\right|$, signaling the presence of entanglement due to a negative eigenvalue. So, the negativity for pure pair states amounts to $\mathcal{N}(\Psi)=\sum_{i<j}\left|c_{i} c_{j}\right|$, which is a necessary and sufficient measure of entanglement, being zero only for factorizable states.

\section{MIXED STATES}

Mixtures of pure states in the same pair basis, define a large nontrivial subset of the full Hilbert space, described by density matrices depending on $d^{2}-1$ independent parameters. A fundamental property of the negativity is that it represents an entanglement monotone under local operations and classical communication (LOCC) for every mixed state in arbitrary dimension [4, 14. Specifically, $\mathcal{N}(P(\rho)) \leq \mathcal{N}(\rho)$ for an arbitrary LOCC $P(\rho)$. Moreover, given that a mixed state is a convex combination of pure states $\rho_{i}$, the negativity is in general a convex function, i.e. $\mathcal{N}\left(\sum_{i} p_{i} \rho_{i}\right) \leq \sum_{i} p_{i} \mathcal{N}\left(\rho_{i}\right)$, with weights obeying to $\sum_{i} p_{i}=1$ and $p_{i} \geq 0, \forall i$.

For a mixed pair state $\rho_{i j}$, a convex combination of pure states in a pair basis (1), the negativity becomes

$$
\mathcal{N}(\rho)=\sum_{i<j}\left|\rho_{i j}\right|
$$

which turns out to be a good measure of entanglement for our class of states, since $\mathcal{N}$ is convex and vanishes only in absence of off-diagonal terms of $\rho_{i j}$, i.e. for factorizable states. In other words, this proves that a necessary and sufficient condition for having entanglement is the non vanishing of negativity, a property which is not valid for general states. In addition, the monotonicity of $\mathcal{N}(\rho)$ introduces a ordering in terms of entanglement content. Despite its simplicity, Eq. 3. constitutes an important result, which may reveal of great utility in the evaluation of entanglement in several systems expressable in a pair basis. Along the same line, one can compute the logarithmic negativity $E_{\mathcal{N}}(\rho) \equiv \log \left\|\rho^{T_{A}}\right\|_{1}=\log \left(1+2 \sum_{i<j}\left|\rho_{i j}\right|\right)$, which bounds the distillable entanglement of $\rho$ [14].

\section{ENTANGLEMENT OF FORMATION}

The EOF $E_{f}(\rho)$ is in general defined as the convex roof

$$
E_{f}(\rho)=\min _{\left\{\mathrm{p}_{\mathrm{k}}, \psi_{\mathrm{k}}\right\}} \sum_{k=1}^{d} p_{k} S^{(d)}\left(\psi_{k}\right) .
$$

that gives the minimum average entropy over all possible decompositions of $\rho=\sum_{k} p_{k}\left|\psi_{k}\right\rangle\left\langle\psi_{k}\right|$ into pure states $\left|\psi_{k}\right\rangle, k=1, \ldots d$, with weights $p_{k}$. The calculation of 
(4) for general states is notoriously a formidable task. However, for our class of states we are able to establish some tight lower bounds of $E_{f}$ of evident use in a variety of applications.

In our case, the task is somewhat facilitated by the crucial property that the element $\left|\psi_{k}\right\rangle$ of every decomposition must also be restricted to the same pair basis as $\rho$. In fact, the diagonal elements $\langle i j|\rho| i j\rangle=0$ for $i \neq j$, can only result from a convex combination of zero diagonal elements $\left\langle i j\left|\rho_{k}\right| i j\right\rangle=0$ for any $k$, since they cannot be negative by definition, as well as the weigths $p_{k}$. As a consequence, even the off-diagonal elements of $\rho_{k}$ that lie outside the pair basis are zero.

The entanglement of any pure state in Eq. (1) is determined by $d-1$ parameters, the Schmidt weights $\mu_{j}=\left|c_{j}\right|$, plus the normalization condition. As known, these coefficients cannot be inferred by the partial trace in the mixed case. Instead, we want to relate them to the offdiagonal entries of $\rho_{i j}$. After relabeling the states such that $\mu_{1} \geq \mu_{2} \geq \cdots \geq \mu_{d}$ (hence $\Gamma_{1} \geq \Gamma_{2} \geq \cdots \geq \Gamma_{d}$ ), it holds that

$$
\mu_{i}^{2}=\frac{1}{2}\left(1-\epsilon_{i} \sqrt{1-4 \Gamma_{i}^{2}}\right)
$$

where

$$
\Gamma_{i}^{2}=\mu_{i}^{2}\left(1-\mu_{i}^{2}\right)=\sum_{j \neq i} \mu_{i}^{2} \mu_{j}^{2}=\sum_{j \neq i}\left|\rho_{i j}\right|^{2}
$$

and all $\epsilon_{i}=1$ except $\epsilon_{1}=-1$ if $\left|\mu_{1}\right|^{2}>1 / 2$. Of course, for pure states the quantities $\left|\rho_{i j}\right|^{2}$ are overdetermined, so there are many ways to take $d-1$ of them which are independent. One way to avoid such an overdetermination is to consider only the first row of the density matrix, obtaining $\mu_{j}^{2}=\left|\rho_{1 j}\right|^{2} / \mu_{1}^{2}, j=2, \ldots, d$, and $\mu_{1}^{2}$ as in Eq. (5). Now, we are able to find a lower bound to the EOF, by means of the following:

Theorem For every pair state described by a density matrix $\rho$ in the relabeled basis with $\Gamma_{1} \geq \Gamma_{2} \geq \cdots \geq \Gamma_{d}$, it holds

$$
E_{f}(\rho) \geq F(\mathbf{x}) \equiv-\sum_{i=1}^{d} \alpha_{i}^{2}(\mathbf{x}) \log \alpha_{i}^{2}(\mathbf{x})
$$

where

$$
\alpha_{1}^{2}=\frac{1}{2}\left(1+\sqrt{1-4|\mathbf{x}|^{2}}\right) ; \quad \alpha_{i}^{2}=\frac{\left|x_{i}\right|^{2}}{\alpha_{1}^{2}}, i=2, \ldots, d
$$

and the components of the vector $\mathbf{x} \equiv\left\{\rho_{12}, \rho_{13}, \ldots, \rho_{1 d}\right\}$ are the $d-1$ off-diagonal elements in the first row of $\rho$, which act as independent parameters.

Proof Let us assume that there exist an optimal decomposition of $\rho=\sum_{k} p_{k}\left|\psi_{k}\right\rangle\left\langle\psi_{k}\right|$ formed by an ensemble of pure states $\left\{p_{k},\left|\psi_{k}\right\rangle\right\}$, where each $\psi_{k}$ must belong to the same pair basis set, as discussed above. To any state $\psi_{k}$ of the decomposition, we associate a set of vectors $\mathbf{x}^{k}$ and exact Schmidt weights $\left\{\mu_{i}^{2}\left(\psi_{k}\right)\right\}_{i=1}^{d}$, as given by Eqs.(5) and (6). The off-diagonal elements of $\rho$ contained in $\mathbf{x}$ are given by the convex sum $\mathbf{x}=\sum_{k} p_{k} \mathbf{x}^{k}$. It follows that the EOF is lower bounded by $F(\mathbf{x})$ because

$$
\begin{aligned}
E_{f}(\rho) & =-\sum_{k} p_{k}\left(\sum_{i=1}^{d} \mu_{i}^{2}\left(\psi_{k}\right) \log \mu_{i}^{2}\left(\psi_{k}\right)\right) \\
& \geq-\sum_{k} p_{k}\left(\sum_{i=1}^{d} \alpha_{i}^{2}\left(\mathbf{x}^{k}\right) \log \alpha_{i}^{2}\left(\mathbf{x}^{k}\right)\right) \\
& =\sum_{k} p_{k} F\left(\mathbf{x}^{k}\right) \geq F(\mathbf{x}) .
\end{aligned}
$$

The first inequality has been obtained by observing that $\forall z \in[0,1]$, we get $\frac{1}{2}(1+\sqrt{1-z}) \log \left[\frac{1}{2}(1+\sqrt{1-z})\right] \geq$ $\frac{1}{2}(1-\sqrt{1-z}) \log \left[\frac{1}{2}(1-\sqrt{1-z})\right]$, that gives a lower bound for the first term in the sum in (9), and eliminates the problem of determining the sign $\epsilon_{1}$. This latter inequality is also crucial to compensate the increase of the second term in the sum, when $e^{-1} \leq \mu_{1}^{2} \leq \mu_{2}^{2} \leq 1 / 2$. The second inequality in (9) holds thanks to the convexity of $F(\mathbf{x})$ over its domain $\left\{\mathbf{x}:|\mathbf{x}|^{2} \in\left[0, \frac{1}{4}\right]\right\}$. After observing that $F$ depends only on the moduli of the components of $\mathbf{x}=\left\{x_{i}\right\}_{i=2}^{d}$, we get

$$
\begin{aligned}
\sum_{k} p_{k} F\left(\mathbf{x}^{k}\right) & =\sum_{k} p_{k} F\left(\left\{\left|x_{i}^{k}\right|\right\}\right) \geq F\left(\left\{\sum_{k} p_{k}\left|x_{i}^{k}\right|\right\}\right) \\
& \geq F\left(\left\{\left|\sum_{k} p_{k} x_{i}^{k}\right|\right\}\right)=F(\mathbf{x}),
\end{aligned}
$$

where the first inequality comes from the convexity of $F(\mathbf{v})$ as a function of a real positive vector $\mathbf{v}$, such that $|\mathbf{v}|^{2} \in\left[0, \frac{1}{4}\right]$, while the second inequality is a consequence of the triangular inequality $|z+w| \leq|z|+|w|$, with $z, w \in \mathbb{C}$ and the monotonicity of $F(\mathbf{v})$ with respect to any of its components. The latter property can be directly proven by calculating $\nabla[F(\mathbf{x})]$ and showing that all its components are non negative for $|\mathbf{v}|^{2} \in\left[0, \frac{1}{4}\right]$ (see Appendix A). Proving that $F(\mathbf{v})$ is convex for any $d$ by checking directly the positiveness of its Hessian $\mathcal{H}[F(\mathbf{v})]$ can be a hard task. By means of a mathematical stratagem we have found a way to write Eq. (7) as a sum of convex functions $F(\mathbf{v})=\sum_{k} F_{k}(\mathbf{v})$, where each $F_{k}$ explicitly depends only on the two variables $|\mathbf{v}|$ and $v_{k}$, whose convexity is analytically proven through ordinary calculus methods (see Appendix A). This shows that $F(\mathbf{v})$ is indeed convex in every dimension.

A step further in the seek of lower bounds for the EOF can be made by defining the function $G\left(\mathbf{x}_{1}, \ldots, \mathbf{x}_{d}\right)$ in a similar way as $F(\mathbf{x})$ in Eqs. (7) and (8), but with

$$
\begin{aligned}
& \alpha_{1}^{2}=\left(1+\sqrt{1-4\left|\mathbf{x}_{1}\right|^{2}}\right) / 2, \\
& \alpha_{i}^{2}=\left(1-\sqrt{1-4\left|\mathbf{x}_{i}\right|^{2}}\right) / 2, \quad i=2, \ldots, d
\end{aligned}
$$

where $\mathbf{x}_{i} \equiv\left\{\rho_{i i^{\prime}}, i^{\prime} \neq i\right\}$. In this new definition the number of independent variables is increased to $d(d-1) / 2$. Unfortunately, the function $G$ is not convex over all its domain, a property that would be a sufficient condition 

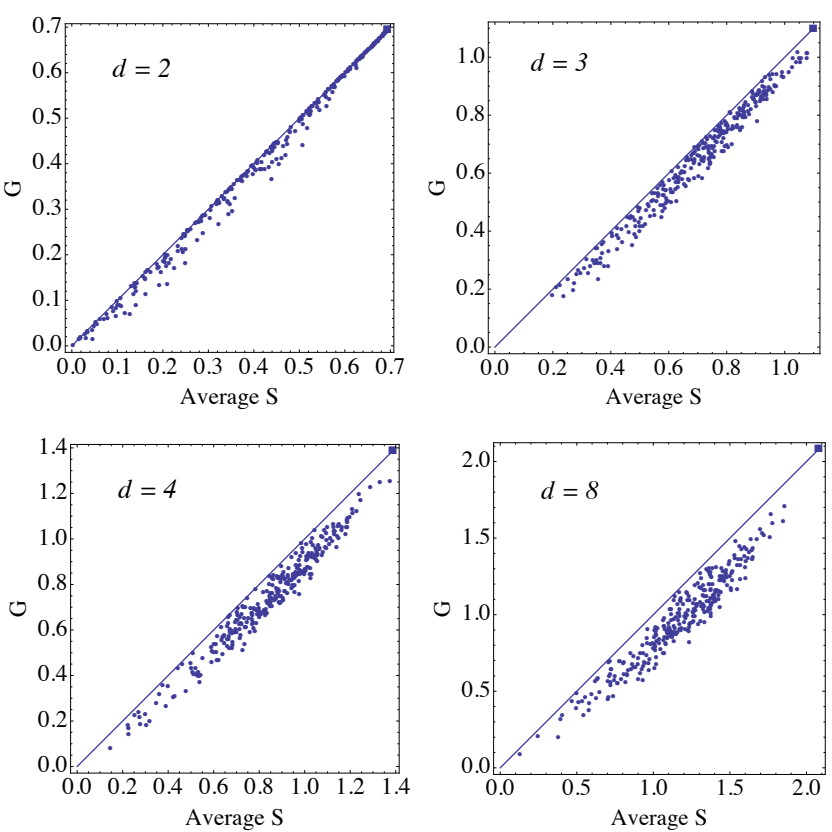

Figure 1: (Color online) The average entropy (horizontal axis) is plotted against the function $G$ (vertical axis) for several density matrices $\rho$ in various dimensions $d$. Each point is calculated from a set of randomly generated pure states, combined with random weights (see text). The fact that all the points lie below the bisector line, constitutes a stochastic demonstration that $G$ is a lower bound to the EOF.

for proving its validity as a lower bound for EOF, like in the previous theorem. However, strictly speaking we need only the weaker property of convexity with respect to the set of pure states (as opposed to the overall set of density matrices). This latter feature is indeed displayed by $G$, though it seems to be rather hard to prove it analytically. Instead, we provide a stochastic demonstration by generating several samples of $\rho$ through random mixtures of pure states $\left\{p_{k}, \psi_{k}\right\}$ with uniformly distributed $p_{k}$ and coefficients of $\psi_{k}$. We have numerically checked that the average entropy $\sum_{k} p_{k} S^{(d)}\left(\psi_{k}\right)$ of every sampled decomposition is always larger than the lower bound $G$, calculated directly from the matrix elements of $\rho$. Without loss of generality, we have generated pure states of the form (1) with real positive coefficients, i.e. pure state decompositions which are closer to the optimal one (that we do not know) thank to the triangular inequality and because the entropy depends only on the absolute value of the entries of $\rho$. The results of the numerical simulations are collected in Fig,1.

Finally, we consider the lower bound introduced in Ref. 18, that depends on $\rho$ only through one variable: the maximum between the partial transpose and the realignment, for general states. Restricting to pair states, this single parameter reduces to the negativity. In the set of pure states, there is no unique correspondence between negativity and entropy for $d>2$, as there can be states with the same negativity but different entropy.
However, one may introduce a convex function $s(\mathcal{N})$, that for any $\mathcal{N}$ is not larger than the minimum entropy $S_{\text {min }}^{(d)}(\mathcal{N})=\min _{\left\{\psi_{\mathrm{k}}, \mathcal{N}\right\}} S^{(d)}\left(\psi_{k}\right)$ in the manifold of all pure states $\left|\psi_{k}\right\rangle$ with a given negativity $\mathcal{N}$. This optimization problem has been solved in [25], with solution

$s(\mathcal{N})= \begin{cases}H_{2}(\gamma)+(1-\gamma) \log (d-1), & \mathcal{N} \in\left[0, \frac{3}{2}-\frac{2}{d}\right] \\ \frac{2 \mathcal{N}+1-d}{d-2} \log (d-1)+\log d, & \mathcal{N} \in\left[\frac{3}{2}-\frac{2}{d}, \frac{d-1}{2}\right]\end{cases}$

where $\gamma(\mathcal{N})=\frac{1}{d^{2}}[\sqrt{2 \mathcal{N}+1}+\sqrt{(d-1)(d-2 \mathcal{N}-1)}]^{2}$. By assuming first to know the optimal decomposition $\left\{p_{k},\left|\psi_{k}\right\rangle\right\}$ that gives the minimum in Eq. (4), one can apply the inequalities

$$
\begin{aligned}
E_{f}(\rho) & \geq \sum_{k} p_{k} \min _{\left\{\mathrm{p}_{\mathrm{k}}, \psi_{\mathrm{k}}\right\}} S^{(d)}\left(\psi_{k}\right) \geq \sum_{k} p_{k} s\left(\mathcal{N}\left(\psi_{k}\right)\right) \\
& \geq s\left(\sum_{k} p_{k} \mathcal{N}\left(\psi_{k}\right)\right) \geq s(\mathcal{N}(\rho))
\end{aligned}
$$

thanks to the convexity of both $s$ and $\mathcal{N}$. It is clear that the function $s(\mathcal{N}(\rho))$ sets a lower bound to the EOF of $\rho$. The advantage of introducing the function $s$ is to establish a 1-1 relationship between negativity and EOF, like in the two-qubit case. On the one hand, this lower bound to EOF is exact for isotropic states [25] and in our case works very well for states where the off-diagonal terms assume very similar values. On the other hand, $F$ and $G$ set better lower bounds for states where few $\Gamma_{j}$ 's dominate over the others. In particular, in large dimensions when $d \gg \mathcal{N}$, the leading term $s(\mathcal{N}) \approx(2 \mathcal{N}-$ 1) $d^{-1} \log d$ goes to zero. In fact, in infinite dimension $s(\mathcal{N})$ fails to give a reliable lower bound for every finite $\mathcal{N}$, while $F$ and $G$ still give a good estimation of EOF, e.g. in the case of two-mode squeezed states (see Appendix B].

The best estimation of the EOF for an arbitrary state $\rho$ is given by $\max \{F(\rho), G(\rho), s(\rho)\}$. In Fig 2 we show a comparative plot of the three lower bounds $F, G$, and $s$ for some randomly generated states (with the sampling method used for Fig.1), ordered according to their negativity. As expected from the previous analysis, $s$ is a good estimation of EOF for some instances in low dimension and close to the maximally entangled state. However, as the dimension is increased $s$ becomes useless, while $F$ and $G$ give sizable estimates. For high dimensions, we observe that $G$ is slightly greater than $F$, so it tends to dominate, as shown in Fig 2 for $d=20$, where the square symbols often overtake the circles. More detailed applications of entanglement estimation of states in pair basis will be considered in forthcoming works.

\section{Conclusions}

In this article we have significantly extended the family of states for which the negativity is a necessary and sufficient condition for entanglement by considering mixtures of pure states written in the paired form (1), which 

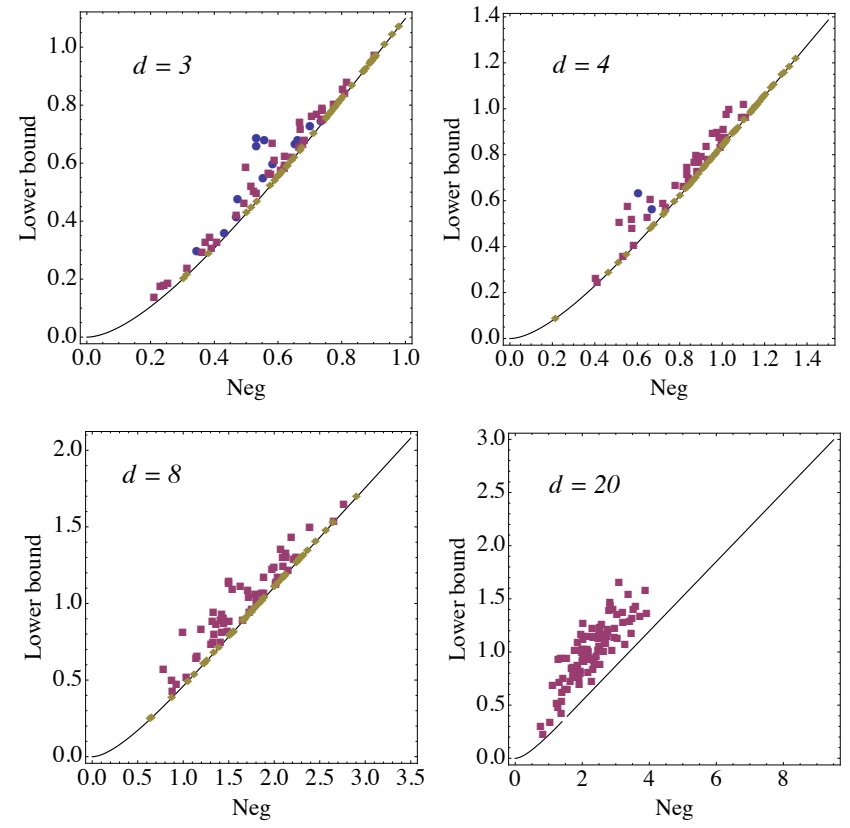

Figure 2: (Color online) The maximum between the lower bounds to the EOF in various dimensions $d$. Circles (blue), squares (red) and rhombs (yellow) represent $F(\rho), G(\rho)$ and $s(\rho)$, respectively. The sample states $\rho$ are arranged according to their negativity $\mathcal{N}$ (horizontal axis). For reference, we have plotted the curve $s(\mathcal{N})$.

are relevant in several physical situations. We have also found new lower bounds improving the estimation of the EOF with respect to other quantities known in the literature. We believe that our scheme for determining the functions $F$ and $G$ may be extended to arbitrary states, shining some light toward the identification of a general efficient entanglement measure.

\section{Acknowledgements}

We acknowledge EU-ERC project no. 267915 (OPTINF), the Compagnia di San Paolo and NATO grant SFP 984397 for partial support.

\section{Appendix A: Convexity of the function $F$}

The entropy function $F$, defined in Eq. (7),

$$
F(\mathbf{x}) \equiv-\sum_{i=1}^{d} \alpha_{i}(\mathbf{x}) \log \alpha_{i}(\mathbf{x})
$$

is notoriously a concave function as a function of the $\alpha$ 's. However, here we want to proof that $F$ as a function of the $(d-1)$-dimensional vector $\mathbf{x} \equiv\left\{x_{i}, i=1, \ldots, d-1\right\}$ is instead convex over the domain $\mathcal{D}=\left\{x_{i} \geq 0,|\mathbf{x}|^{2} \leq\right.$ $1 / 4\}$, with the choice

$$
\alpha_{1}=\frac{1}{2}\left(1+\sqrt{1-4 r^{2}}\right) ; \quad \alpha_{i}=\frac{x_{i-1}^{2}}{\alpha_{1}}, i=2, \ldots, d
$$

where we have renamed $r=\sqrt{|\mathbf{x}|^{2}}$.

Prooving the convexity of $F$ in every dimension $d$ through a "brute force" demonstration of the positiveness of its Hessian, can be a hard task. Here instead we proceed by presenting a detailed calculation after splitting the function in a sum of convex terms.

For convenience, we rewrite $F(\mathbf{x})$ as

$$
\begin{aligned}
F= & -\alpha_{1} \log \alpha_{1}-\sum_{i=1}^{d-1} \frac{x_{i}^{2}}{\alpha_{1}} \log \frac{x_{i}^{2}}{\alpha_{1}} \\
= & \left(1-2 \alpha_{1}\right) \log \alpha_{1}-\frac{1}{\alpha_{1}}\left(\sum_{i=1}^{d-1} x_{i}^{2} \log \frac{x_{i}^{2}}{r^{2}}-r^{2} \log r^{2}\right) \\
= & \sum_{k=1}^{d-1} \frac{x_{k}^{2}}{r^{2}}\left[-\alpha_{1} \log \alpha_{1}-\left(1-\alpha_{1}\right) \log \left(1-\alpha_{1}\right)\right. \\
& \left.-\left(1-\alpha_{1}\right) \log \frac{x_{k}^{2}}{r^{2}}\right] \equiv \sum_{k=1}^{d-1} F_{k}(\mathbf{x})
\end{aligned}
$$

where we have used the identity $r^{2}=\alpha_{1}\left(1-\alpha_{1}\right)$. We have rewritten $F$ in this way because it is feasible to show that $F_{k}(\mathbf{x})$ is convex in $\mathcal{D}$ for every $k=1, \ldots, d-1$. Since the sum of convex functions is convex, this will prove our statement.

Lemma The function

$$
F_{k}(\mathbf{x})=\frac{x_{k}^{2}}{r^{2}}\left[H_{C}(r)-f(r) \log \frac{x_{k}^{2}}{r^{2}}\right]
$$

where

$$
\begin{aligned}
H_{C}(r)= & -\frac{1}{2}\left(1+\sqrt{1-4 r^{2}}\right) \log \left[\frac{1}{2}\left(1+\sqrt{1-4 r^{2}}\right)\right] \\
& -\frac{1}{2}\left(1-\sqrt{1-4 r^{2}}\right) \log \left[\frac{1}{2}\left(1-\sqrt{1-4 r^{2}}\right)\right] \\
f(r)= & \frac{1}{2}\left(1-\sqrt{1-4 r^{2}}\right)
\end{aligned}
$$

is convex in the domain $\mathcal{D}$ and it is an increasing function with respect to any component of $\mathbf{x}$. (Notice that $H_{C}$ has just the same form as the entropy for a pair of qubits as a function of the concurence)

Proof We use the fact that $F_{k}(\mathbf{x})$ depends explicitly only on the two quantities $g_{1}(\mathbf{x})=|\mathbf{x}|$ and $g_{2}(\mathbf{x})=x_{k} / r$, thus

$$
\begin{aligned}
F_{k}(\mathbf{x}) & =F_{k}\left(g_{1}(\mathbf{x}), g_{2}(\mathbf{x})\right) \\
& \equiv g_{2}^{2}(\mathbf{x})\left[H_{C}\left(g_{1}(\mathbf{x})\right)-f\left(g_{1}(\mathbf{x})\right) \log g_{2}^{2}(\mathbf{x})\right]
\end{aligned}
$$

whose gradient and Hessian functions in terms of $\mathbf{g}=$ $\left(g_{1}, g_{2}\right)$ are expressed as 


$$
\begin{gathered}
\nabla_{g}\left[F_{k}(\mathbf{g})\right]=\left(G_{10}, G_{01}\right)=\left(g_{2}^{2}\left(H_{C}^{\prime}-f^{\prime} \log g_{2}^{2}\right), 2 g_{2}\left[H_{C}-f\left(1+\log g_{2}^{2}\right)\right]\right) \\
\mathcal{H}_{g}\left[F_{k}(\mathbf{g})\right]=\left(\begin{array}{ll}
G_{20} & G_{11} \\
G_{11} & G_{02}
\end{array}\right)=\left(\begin{array}{cc}
g_{2}^{2}\left(H_{C}^{\prime \prime}-f^{\prime \prime} \log g_{2}^{2}\right) & 2 g_{2}\left[H_{C}^{\prime}-f^{\prime}\left(1+\log g_{2}^{2}\right)\right] \\
2 g_{2}\left[H_{C}^{\prime}-f^{\prime}\left(1+\log g_{2}^{2}\right)\right] & 2\left[H_{C}-f\left(3+\log g_{2}^{2}\right)\right]
\end{array}\right)
\end{gathered}
$$

where the primes denote derivation of functions of one variable with respect to their argument. For brevity, we denote $G_{n m}=\partial_{g 1}^{n} \partial_{g 2}^{m} F_{k}\left(g_{1}, g_{2}\right)$.

The gradient in terms of the original coordinates, takes the form

$$
\begin{aligned}
\nabla F_{k} & =\frac{\partial F_{k}}{\partial x_{j}}=\sum_{l} \frac{\partial F_{k}}{\partial g_{l}} \frac{\partial g_{l}}{\partial x_{j}} \\
& =\sum_{l} \nabla_{g}\left[F_{k}(\mathbf{g})\right]_{l} \mathcal{J}[\mathbf{g}(\mathbf{x})]_{l j}=\nabla_{g}[F(\mathbf{g})] \cdot \mathcal{J}[\mathbf{g}(\mathbf{x})] \\
& =G_{10} \nabla g_{1}+G_{01} \nabla g_{2}
\end{aligned}
$$

The Jacobian of the vector function $\mathbf{g}(\mathbf{x})$ is defined as $\mathcal{J}[\mathbf{g}(\mathbf{x})]_{i j}=\frac{\partial g_{i}}{\partial x_{j}}$. The Hessian is

$$
\begin{aligned}
\mathcal{H} F_{k}= & \mathcal{J}\left[\nabla F_{k}\right]=\left[\frac{\partial^{2} F_{k}}{\partial x_{i} \partial x_{j}}\right]_{i j}=\frac{\partial}{\partial x_{i}}\left(\sum_{l} \frac{\partial F_{k}}{\partial g_{l}} \frac{\partial g_{l}}{\partial x_{j}}\right) \\
= & \sum_{l}\left\{\sum_{l^{\prime}}\left(\frac{\partial F_{k}}{\partial g_{l} \partial g_{l^{\prime}}} \frac{\partial g_{l^{\prime}}}{\partial x_{i}} \frac{\partial g_{l}}{\partial x_{j}}\right)+\frac{\partial F_{k}}{\partial g_{l}} \frac{\partial^{2} g_{l}}{\partial x_{i} \partial x_{j}}\right\} \\
= & \sum_{l} \sum_{l^{\prime}}\left(\mathcal{J}[\mathbf{g}(\mathbf{x})]_{l j} \mathcal{H}_{g}\left[F_{k}(\mathbf{g})\right]_{l l^{\prime}} \mathcal{J}[\mathbf{g}(\mathbf{x})]_{l^{\prime} j}\right) \\
& +\sum_{l} \nabla_{g}\left[F_{k}(\mathbf{g})\right]_{l} \mathcal{H}\left[g_{l}(\mathbf{x})\right]_{i j} \\
= & \mathcal{J}[\mathbf{g}(\mathbf{x})]^{T} \mathcal{H}_{g}\left[F_{k}(\mathbf{g})\right] \mathcal{J}[\mathbf{g}(\mathbf{x})]+\nabla_{g}\left[F_{k}(\mathbf{g})\right] \mathcal{H}[\mathbf{g}(\mathbf{x})] \\
= & G_{20} \nabla g_{1} \otimes \nabla g_{1}+G_{02} \nabla g_{2} \otimes \nabla g_{2} \\
& +G_{11}\left(\nabla g_{2} \otimes \nabla g_{1}+\nabla g_{1} \otimes \nabla g_{2}\right) \\
& +G_{10} \mathcal{H}\left(g_{1}\right)+G_{01} \mathcal{H}\left(g_{2}\right)
\end{aligned}
$$

Explicitly,

$$
\begin{aligned}
& {\left[\nabla g_{1}\right]_{i}=\frac{\partial}{\partial x_{i}} r=\frac{\partial}{\partial x_{i}} \sqrt{\sum_{j} x_{j}^{2}}=\frac{x_{i}}{r}} \\
& {\left[\nabla g_{2}\right]_{i}=\frac{\partial}{\partial x_{i}} \frac{x_{k}}{r}=\frac{1}{r^{3}}\left(r^{2} \delta_{i k}-x_{k} x_{i}\right)}
\end{aligned}
$$

These two vectors are othogonal: another good feature of decomposing $F$ in the form of Eq. A3.

From Eq. A5 it is immediate to verify that $\partial_{x_{j}}\left[F_{k}(\mathbf{x})\right] \geq 0, \forall j$, because $G_{10}$ and $G_{01}$ are both non negative in $\mathcal{D}$ as well as each component of $\nabla g_{1}$ and $\nabla g_{2}$. Since each component of the gradient $\nabla[F(\mathbf{x})]$ is a sum of positive contributions, it follows that $F(\mathbf{x})$ is an increasing function with respect to each component of $\mathbf{x}$.
The outer products of derivative terms are

$$
\begin{aligned}
& {\left[\nabla g_{1} \otimes \nabla g_{1}\right]_{i j}=\frac{x_{i} x_{j}}{r^{2}}} \\
& {\left[\nabla g_{2} \otimes \nabla g_{1}\right]_{i j}=\frac{1}{r^{4}}\left(r^{2} \delta_{i k}-x_{k} x_{i}\right) x_{j}=\left[\nabla g_{1} \otimes \nabla g_{2}\right]_{j i}} \\
& {\left[\nabla g_{2} \otimes \nabla g_{2}\right]_{i j}=\frac{1}{r^{6}}\left(r^{2} \delta_{i k}-x_{k} x_{i}\right)\left(r^{2} \delta_{j k}-x_{k} x_{j}\right)}
\end{aligned}
$$

and

$$
\begin{aligned}
{\left[\mathcal{H} g_{1}\right]_{i j}=} & \mathcal{J}\left[\nabla g_{1}\right]_{i j}=\frac{\delta_{i j}}{r}-\frac{x_{i} x_{j}}{r^{3}}=\frac{1}{r}\left(\mathbb{I}-\nabla g_{1} \otimes \nabla g_{1}\right) \\
{\left[\mathcal{H} g_{2}\right]_{i j}=} & \mathcal{J}\left[\nabla g_{2}\right]_{i j}=\frac{\partial}{\partial x_{j}}\left[\frac{1}{r^{3}}\left(r^{2} \delta_{i k}-x_{k} x_{i}\right)\right] \\
= & -\frac{1}{r}\left(\nabla g_{2} \otimes \nabla g_{1}+\nabla g_{1} \otimes \nabla g_{2}\right) \\
& -\frac{x_{k}}{r^{3}}\left(\mathbb{I}-\nabla g_{1} \otimes \nabla g_{1}\right)
\end{aligned}
$$

Thus the Hessian becomes

$$
\begin{aligned}
\mathcal{H} F_{k}= & \frac{1}{r^{3}}\left(x_{k} G_{01}-r^{2} G_{10}+r^{3} G_{20}\right) \nabla g_{1} \otimes \nabla g_{1} \\
& +\left(G_{11}-\frac{1}{r} G_{01}\right)\left(\nabla g_{2} \otimes \nabla g_{1}+\nabla g_{1} \otimes \nabla g_{2}\right) \\
& +G_{02} \nabla g_{2} \otimes \nabla g_{2}+\frac{1}{r^{3}}\left(r^{2} G_{10}-x_{k} G_{01}\right) \mathbb{I}
\end{aligned}
$$

This matrix can be decomposed in direct sum of a non singular $2 \times 2$ matrix written in the basis $\left\{\nabla g_{1}, \nabla g_{2}\right\}$ and a uniform diagonal part. The square norm of $\nabla g_{2}$ is

$$
\left\|\nabla g_{2}\right\|^{2}=\frac{1}{r^{4}}\left(r^{2}-x_{k}^{2}\right)=\frac{1}{r^{2}}\left(1-g_{2}^{2}\right)
$$

Introducing the orthonormal basis $b_{1}=\nabla g_{1}$ and $b_{2}=$ $\nabla g_{2} /\left\|\nabla g_{2}\right\|$, we obtain

$$
\begin{aligned}
\mathcal{H} F_{k}= & \frac{1}{r^{2}}\left(g_{2} G_{01}-r G_{10}+r^{2} G_{20}\right) b_{1} \otimes b_{1} \\
& +\frac{1-g_{2}^{2}}{r}\left(G_{11}-\frac{1}{r} G_{01}\right)\left(b_{1} \otimes b_{2}+b_{2} \otimes b_{1}\right) \\
& +\frac{1-g_{2}^{2}}{r^{2}} G_{02} b_{2} \otimes b_{2}+\frac{1}{r^{2}}\left(r G_{10}-g_{2} G_{01}\right) \mathbb{I}
\end{aligned}
$$

In the two dimensional subspace spanned by $b_{1}$ and $b_{2}$, the Hessian becomes

$$
\mathcal{H}_{\|} F_{k}=\left(\begin{array}{cc}
\alpha & \beta \\
\beta & \gamma
\end{array}\right)
$$


where

$$
\begin{aligned}
\alpha & =G_{20} \\
\beta & =\frac{\sqrt{1-g_{2}^{2}}}{r}\left(G_{11}-\frac{1}{r} G_{01}\right) \\
\gamma & =\frac{1-g_{2}^{2}}{r^{2}} G_{02}+\frac{1}{r^{2}}\left(r G_{10}-g_{2} G_{01}\right)
\end{aligned}
$$

In the complementary space, we simply have

$$
\mathcal{H}_{\perp} F_{k}=\eta\left(\mathbb{I}-b_{1} \otimes b_{1}-b_{2} \otimes b_{2}\right)
$$

with $\eta=\frac{1}{r^{2}}\left(r G_{10}-g_{2} G_{01}\right)$.

Using the Sylvester's criterion for testing the positivity of matrices, we conclude that $F_{k}$ is convex if $\eta$, $\alpha$, and $\alpha \gamma-\beta^{2}$ are all non negative. These are continuous functions of two variables defined in the set $r \in(0,1 / 2)$ and $g_{2} \in(0,1]$, so their sign can be evaluated throuh standard calculus methods. The matrix element $\alpha=g_{2}^{2}\left(H_{C}^{\prime \prime}-f^{\prime \prime} \log g_{2}^{2}\right)$ is easily verified to be non-negative since $-g_{2}^{2} \log g_{2}^{2} \geq 0$ and both $H_{C}$ and $f$ are convex functions. The quantity

$$
\eta=\frac{g_{2}^{2}}{r^{2}}\left(r H_{C}^{\prime}-2 H_{C}+2 f\right)-\frac{g_{2}^{2}}{r^{2}} \log g_{2}^{2}\left(r f^{\prime}-2 f\right)
$$

is made up of two contributions, both positive; in fact $r f^{\prime}-2 f \geq 0$ and $r H_{C}^{\prime}-2 H_{C}+2 f \geq 0$. This latter inequality requires some analysis. As a matter of fact, we can define the function $p(z)=(2 z-1)\left(r H_{C}^{\prime}-2 H_{C}+\right.$ $2 f)$ and we study it as a function of $z \in[1 / 2,1]$, where $r^{2}=z(1-z)$. We find that $p^{\prime \prime}(z)=0$ has a single root in $z_{1}$ and is increasing: this means that $p^{\prime}(z)$ has a negative minimum in $z_{1}$ and has a zero in $z_{2}<z_{1}$ and $z_{3}=1$. Going back to $p(z)$, we learn that it has a positive maximum in $z_{2}$ and $p(1 / 2)=p(1)=0$. This means that $p(z)$ is nonnegative in its domain, hence also the function $r H_{C}^{\prime}-2 H_{C}+2 f$. This proves that $\eta \geq 0$.

Finally, the determinant

$$
\begin{aligned}
\alpha \gamma-\beta^{2}= & \frac{1}{r^{2}}\left\{\left(1-g_{2}^{2}\right) G_{20} G_{02}+G_{20}\left(r G_{10}-g_{2} G_{01}\right)\right. \\
& \left.-\left(1-g_{2}^{2}\right)\left(G_{11}-\frac{1}{r} G_{01}\right)^{2}\right\}
\end{aligned}
$$

can be efficiently evaluated numerically with arbitrary precision, as it is a continuous function of two variables in the compact domain $r \in(0,1 / 2)$ and $g_{2} \in(0,1]$. An analytic proof in closed form can be performed, in the same fashion as the previous quantities, but it turns out to be rather cumbersome since it involves higher derivatives and very long expressions. In Fig. 3 we show graphically that $\alpha \gamma-\beta^{2} \geq 0$ and in particular we observe that it is monotonically increasing with $r$.

As this proof is valid for every permitted value of $r$ and $x_{k}$, it is shown that every $F_{k}$ in the sum of Eq. A3 is convex. This concludes our proof of convexity of $F(\mathbf{x})$ in Eq. (7).

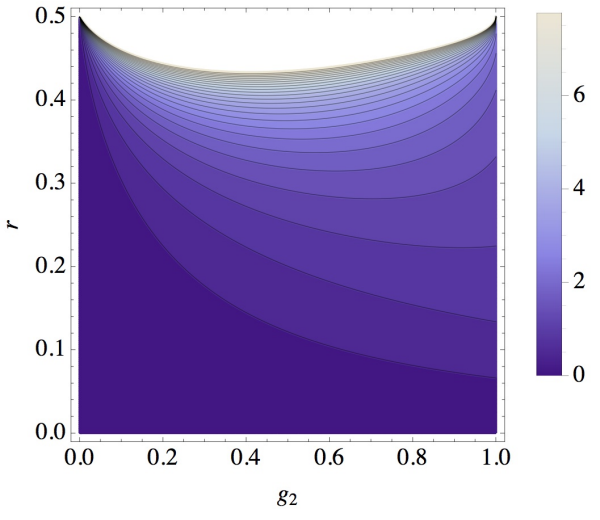

Figure 3: (Color online) The determinant $\alpha \gamma-\beta^{2}$ can be proven to be non negative as a function of its explicit dependence $r^{2}$ and $g_{2}^{2}$.

\section{Appendix B: Test on two-mode squeezed states}

Given a Fock space of two bosonic modes $\left|n_{1}\right\rangle_{A} \otimes\left|n_{2}\right\rangle_{B}$, the pure two-mode squeezed states have the form

$$
\left|\Psi_{r}\right\rangle=\frac{1}{\cosh (r)} \sum_{n=0}^{\infty} \tanh ^{n}(r)|n\rangle_{A} \otimes|n\rangle_{B}
$$

where $r \geq 0$ is the squeezing parameter. Pair states of the form (B1) belong to an infinite dimensional Hilbert space and are a good playground for testing lower bounds to the entanglement of formation (EOF). Furthermore, they are exceptionally interesting for experimental realizations in quantum technologies.

The density matrix $\rho_{i j}=\left\langle j \mid \Psi_{r}\right\rangle\left\langle\Psi_{r} \mid i\right\rangle$ in the pair basis $|i\rangle \equiv|i, i\rangle$ is written as

$$
\rho_{i j}=\left|c_{i} c_{j}\right|=\frac{1}{\cosh ^{2}(r)} \tanh ^{i}(r) \tanh ^{j}(r) .
$$

The negativity for this state can be easily computed, giving $\mathcal{N}\left(\Psi_{r}\right)=e^{r} \sinh (r)$, and is finite for every finite $r$. On the one hand, the entropy can be computed exactly, yielding

$$
\begin{aligned}
S\left(\Psi_{r}\right) & =-\sum_{n=0}^{\infty} c_{n}^{2} \log c_{n}^{2} \\
& =\cosh ^{2}(r) \log \left[\cosh ^{2}(r)\right]-\sinh ^{2}(r) \log \left[\sinh ^{2}(r)\right]
\end{aligned}
$$

On the other hand our lower bound $F$ is given by

$$
\begin{aligned}
F\left(\Psi_{r}\right) & =\cosh ^{2}(r) \log \left[\cosh ^{2}(r)\right]-\sinh ^{2}(r) \log \left[\sinh ^{2}(r)\right] \\
& +\Theta\left(\frac{1}{2}-\frac{1}{\cosh ^{2}(r)}\right)\left\{\cosh ^{-2}(r) \log \left[\cosh ^{-2}(r)\right]\right. \\
& \left.-\tanh ^{2}(r) \log \left[\tanh ^{2}(r)\right]\right\}
\end{aligned}
$$

where $\Theta(x)$ is the theta function of Heaviside. The lower bound given in Ref.[18] is $s(\mathcal{N})=0$, because in infinite dimension we can always find states with finite negativity 


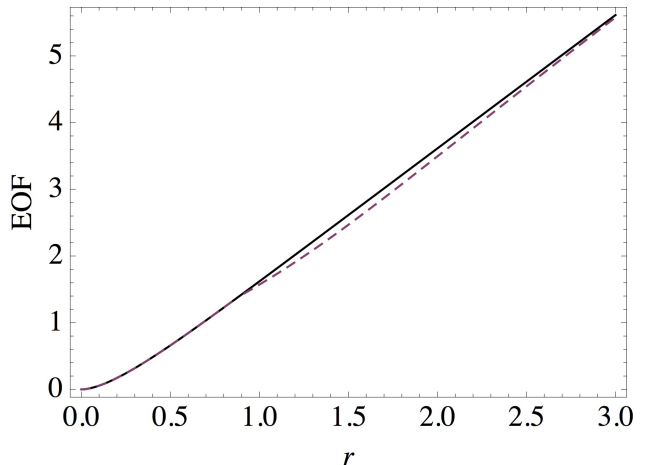

Figure 4: (Color online) The exact entropy $S\left(\Psi_{r}\right)$ for the twomode squeezed state B1 compared with the bound $F\left(\Psi_{r}\right)$ for the entanglement of formation. and zero entropy. In Fig. (4) we can appreciate that in this test case the lower bound $F$ to the EOF gives a very good estimation of the entropy of formation (in this pure case, the entropy).
[1] M. Genovese, Phys. Rep. 413, 319 (2005).

[2] A. Wright, Nature Physics 9, 264 (2013); M. Genovese, Advanced Science Letters 2, 303 (2009).

[3] N. Lambert et al., Nature Physics 9, 10 (2013).

[4] I. Bengtsson and K. Zyczkowski, Geometry of Quantum States (Cambridge University Press, 2006).

[5] M. A. Nielsen and I. L. Chuang, Quantum Computation and Quantum Information (Cambridge University Press, 2000).

[6] L. Amico, R. Fazio, A. Osterloh, and V. Vedral, Rev. Mod. Phys. 80, 517 (2008).

[7] L. Campos Venuti, C. Degli Esposti Boschi, and M. Roncaglia, Phys. Rev. Lett. 96, 247206 (2006); L. Campos Venuti, C. Degli Esposti Boschi, and M. Roncaglia, Phys. Rev. Lett. 99, 060401 (2007).

[8] O. Gühne and G. Tóth, Phys. Rep. 474, 1 (2009).

[9] P. Facchi, G. Florio, and S. Pascazio, Int. J. of Quantum Inf. 5, 219 (2007).

[10] T. Moroder et al., Phys. Rev. Lett. 111, 030501 (2013); D.S. Tasca et al., Phys. Rev. Lett. 110, 210502 (2013); C. Branciard et al., Phys. Rev. Lett. 110, 060405 (2013); Z.-H. Chen et al., Phys. Rev. Lett. 109, 200503 (2013); V. Vedral, Nature 453, 1004 (2013);

[11] W. K. Wootters, Phys. Rev. Lett. 80, 2245 (1998).

[12] M. Horodecki, P. Horodecki, and R. Horodecki, Physics Letters A 223, 1 (1996).

[13] R. F. Werner, Phys. Rev. A 40, 4277 (1989).
[14] G. Vidal and R. F. Werner, Phys. Rev. A 65, 032314 (2002).

[15] A. Anfossi, P. Giorda, A. Montorsi, and F. Traversa, Phys. Rev. Lett. 95, 056402 (2005).

[16] P. Calabrese, J. Cardy, and E. Tonni, Phys. Rev. Lett. 109, 130502 (2012).

[17] R.A. Santos, V. Korepin, S. Bose Phys. Rev. A 84, 062307 (2011).

[18] K. Chen, S. Albeverio, and S.-M. Fei, Phys. Rev. Lett. 95, 210501 (2005).

[19] L. Mandel and E. Wolf, Optical Coherence and Quantum Optics (Cambridge University Press, 1995).

[20] A. Ferraro, S. Olivares, and M. G. A. Paris, Gaussian states in quantum information (Bibliopolis, 2005).

[21] E. D. Lopaeva, I. Ruo Berchera, I. P. Degiovanni, S. Olivares, G. Brida, and M. Genovese, Phys. Rev. Lett. 110, 153603 (2013).

[22] R. M. Gomes et al., Proc. Natl. Acad. Sci. U.S.A. 106, 21517 (2009).

[23] M. Krenn et al., Proc. Natl. Acad. Sci. U.S.A. 111, 6243 (2014).

[24] R. Horodecki, P. Horodecki, M. Horodecki, and K. Horodecki, Rev. Mod. Phys. 81, 865 (2009).

[25] B. M. Terhal and K. G. H. Vollbrecht, Phys. Rev. Lett. 85, 2625 (2000). 